\author{
Chromosomal Population analysis on Cows Descendant \\ FROM A TRANSLOCATION HETEROZYGOTE A.I. BULI, IN BAVARIA \\ M. ENKEN \\ Lehrstuhl für Tierzucht der Technischen Universität München \\ D 8050 Freising-Weihenstephan, B.R.D.
}

Between the many types of chromosomal aberrations, the transloactions of centric fusion type are very interesting, because they result in change of the form and number of chromosomes of one given species. These aberrations were found in several domesticated animals. In doinestic cattle (B. taurus) there are at least four different types of centric fusions distributed among different breeds and in various countries. The most frequent centric fusion is the I/29. This aberration is found with greater frequency between the bests animals used for reproduction. Wether the different types of centric fusion have a positive or negative effect on zootechnical trials is yet unclear, and so also its origin.

In the present work a centric fusion of the I/29 type in a Brown Swiss cattle from Bavaria is described. The centric fusion was found in a very good A.I. bull, and before it was known that it was a I / 29 translocation carrier his semen was used in round 30,000 inseminations. The material studied consists in more than 400 cows who finished at least their first lactation. The parameter studied does not show any important deviations as expected for a normal good reproducer.

\author{
KOPPLUNG ZWISCHEN BIOCHEMISCHEN MARKERGENEN \\ UND LEISTUNGSEIGENSCHAFTEN BEIM Braunvieh \\ D. ZWIAUER \\ Lehrstuhl für Tierzucht der Technischen Universität München \\ D $805^{\circ}$ Freising-Weihenstephan, B.R.D.
}

In Süddeutschland wurde Blut von ca. I.30o Töchtern von 36 F-I Braunvieh/Brown-Swiss Kreuzungsstieren entnommen und auf Io Blutgruppen und 7 biochemische Polymorphismen getestet. Ziel der vorliegenden Arbeit war es, eventuell vorhandene Zusammenhänge zwischen Milch- bzw. Fettleistung und Markergenen nachzuweisen. Die Verwendung von Kreuzungstieren erschien vorteilhaft, da zu erwarten ist, daß gegenüber Reinzuchttieren aufgrund des hoheren Heterozygotiegrades segregierender Leistungsgene, gegebene Unterschiede deutlicher zu Tage treten.

Die Auswertung der Blutgruppen in bezug auf Pleiotropie bzw. Kopplungszusammenhänge zwischen Markergenen und Milch-bzw. Fettleistung mit Hilfe von LSQ-Analysen ergab keine statistisch signifikante Beziehungen.

\title{
SELEKTIONSEXPERIMENT AUF EBERGERUCH
}

\author{
W. ALSING $\left(^{*}\right)$, R. CLAUS $\left(^{*}\right)$, F. PIRCHNER (**) und H. WILLEKE (*) \\ (*) Lehrstuhl für Physiologie der Fortpflanzung und Laktation, \\ D 8050 Freising-Weihenstephan, B.R.D. \\ (**) Lehrstuhl für Tierzucht der Technischen Universität München, \\ D 8050 Freising-Weihenstephan, B.R.D.
}

Im Rahmen eines Selektionsexperimentes auf hohen beziehungsweise niedrigen E,bergeruch wurden aufgrund der Daten der Ausgangspopulation und der I. Generation folgende Ergebnisse berechnet :

I) Die Differenzen zwischen der hoch- und der niederwertigen Linie bei verschiedenem Alter sind signifikant.

2) Die Heritabilitätswerte für Ebergeruch am I70., I90. und 2 Io. Tag sind .68. \pm I I, .47 \pm .23 und .9r \pm .19 .

3) Die genetischen Korrelationen zwischen dem Gewicht und dem Ebergeruch sind I.47, .86 und $.7^{8}$ für den $170 .$, 190. und 210 . Tag. 